\title{
Robust Synchronization Controller Design for a Class of Uncertain Fractional Order Chaotic Systems
}

\author{
Lin Wang ${ }^{1}$ and Chunzhi Yang ${ }^{2}$ \\ ${ }^{1}$ School of Science, Anhui University of Science and Technology, Huainan 232001, China \\ ${ }^{2}$ Department of Applied Mathematics, Huainan Normal University, Huainan 232038, China \\ Correspondence should be addressed to Chunzhi Yang; yangchunzhi6@163.com
}

Received 30 September 2015; Accepted 29 October 2015

Academic Editor: Guoqiang $\mathrm{Hu}$

Copyright (c) 2016 L. Wang and C. Yang. This is an open access article distributed under the Creative Commons Attribution License, which permits unrestricted use, distribution, and reproduction in any medium, provided the original work is properly cited.

\begin{abstract}
Synchronization problem for a class of uncertain fractional order chaotic systems is studied. Some fundamental lemmas are given to show the boundedness of a complicated infinite series which is produced by differentiating a quadratic Lyapunov function with fractional order. By using the fractional order extension of the Lyapunov stability criterion and the proposed lemma, stability of the closed-loop system is analyzed, and two sufficient conditions, which can enable the synchronization error to converge to zero asymptotically, are driven. Finally, an illustrative example is presented to confirm the proposed theoretical results.
\end{abstract}

\section{Introduction}

As a branch of mathematics, fractional calculus mainly tackles the generalization of the concepts of derivation and integration with arbitrary orders. Although the fractional calculus has been given 300 years ago, its applications to engineering have begun just in recent three decades [1]. Among the literatures studied on fractional order systems, some subjects are studied, for instance, system stability analysis $[2,3]$, chaotic systems synchronization [4-9], optimal control [10], adaptive control [11, 12], finite-time stability analysis $[13,14]$, systems identification [15], applications in finances, and sliding modes.

The synchronization of chaotic systems problem has become more and more attractive to researchers due to its potential applications in secure communication [4-6, 1620]. Reference [20] is the first literature studied on the synchronization between two fractional order chaotic systems, in which the authors indicated that fractional order chaotic systems can also be synchronized by using the similar method as that of their integer counterparts. Meantime, the actual systems are often subject to system uncertainties and external disturbances [21-25]. These uncertainties and disturbances, which have to be considered for analyzing and controlling the system, can be brought through various forms. There have been some results about the stability of fractional order systems with system uncertainties [26-30]. For example, the stability problem of fractional order linear uncertain systems expressed in the transfer function form and the statespace form is, respectively, studied in [27] and [28]. The robust stability and stabilization of fractional order linear systems with positive real uncertainty are investigated in [26] by means of LMI technique. Synchronization for chaotic and uncertain Duffing-Holmes systems has been done by using the sliding mode control method, and fractional order mathematics is used to express the system and sliding mode for synchronization [29]. In [30], synchronization for a class of uncertain fractional order neural networks subject to external disturbances and disturbed system parameters is studied. But it should be pointed out that, in the above literatures, the stability of the closed-loop system is analyzed by using the integer-order Lyapunov methods.

However, the lack of extension of the existing robust control methods to fractional order nonlinear uncertain systems is sensible. Besides, to the authors' best knowledge, there are only a few works dealing with the robust synchronization of uncertain fractional order chaotic systems. In [31], synchronization of uncertain Duffing-Holmes system has 
been studied by using the sliding mode control method. In [32], an adaptive sliding mode controller for synchronization of two fractional order chaotic systems with fully unknown system uncertainties and external disturbances is proposed. In the above literatures, the sliding mode control methods are used. The stability analysis is the same as in integerorder systems. Thanks to the works of Li et al. [2], the Lyapunov direct method (also called the Lyapunov second method) has been extended to fractional order nonlinear systems. In the stability analysis of fractional order systems, the Lyapunov function $V(t)=2 x^{T}(t) x(t)$ is often used. The $\alpha$ th-order of $V(t)$ can be given as ${ }_{0}^{C} D_{t}^{\alpha} V(t)=$ $\left({ }_{0}^{C} D_{t}^{\alpha} x(t)\right)^{T} x(t)+x^{T}(t){ }_{0}^{C} D_{t}^{\alpha} x(t)+2 \sum_{k=1}^{\infty}(\Gamma(1+\alpha) / \Gamma(1+k) \Gamma(1-$ $k+\alpha){ }_{0}^{C} D_{t}^{k} x(t){ }_{0}^{C} D_{t}^{\alpha-k} x(t)$. So the stability analysis may be very hard due to the complicated infinite series.

Based on the above discussions, we know that it is still of considerable importance to seek direct systematic approaches for designing robust synchronization controllers for uncertain fractional order chaotic systems. In this paper, a robust controller is proposed to solve the synchronization problem of fractional order chaotic systems with both system uncertainties and norm bounded external disturbances. The fractional order Lyapunov approach is used to analyze the stability of the estimation error system. It ought to be mentioned that (1) the proof of the complicated boundary condition $\left\|\sum_{k=1}^{\infty}(\Gamma(1+\alpha) / \Gamma(1+k) \Gamma(1-k+\alpha)){ }_{0}^{C} D_{t}^{k} x(t){ }_{0}^{C} D_{t}^{\alpha-k} x(t)\right\| \leq a\|x\|$ is given in this paper; (2) the proposed method is very simple for practical applications; (3) the proposed methods enable us to establish a fundamental stability analysis framework in fractional order nonlinear systems.

The remainder of this paper is organized as follows. Section 2 gives mathematical model of the fractional order systems and some preliminaries. In Section 3, the robust synchronization controller is designed and two sufficient conditions are driven. Simulation studies are included in Section 4. Finally, Section 5 concludes this work.

\section{Preliminaries}

The fractional order integrodifferential operator is the extended concept of integer-order integrodifferential operator. The commonly used definitions in the literatures are Grunwald-Letnikov, Riemann-Liouville, and Caputo definitions. Because of the Caputo derivative taking on the same form as integer-order differential on the initial conditions, having well-understood physical meanings and more applications in engineering, we will use this definition in this paper. The lower limit of the fractional calculus is set as 0 in this paper. The fractional order integral with order $\alpha$ can be expressed as

$$
{ }_{0} D_{t}^{-\alpha} f(t)=\frac{1}{\Gamma(\alpha)} \int_{0}^{t}(t-\tau)^{\alpha-1} f(\tau) d \tau .
$$

The Caputo fractional derivative is defined as follows:

$$
{ }_{0}^{C} D_{t}^{\alpha} f(t)=\frac{1}{\Gamma(n-\alpha)} \int_{0}^{t}(t-\tau)^{n-\alpha-1} f^{(n)}(\tau) d \tau
$$

where $\alpha$ is the fractional order, $n-1 \leq \alpha<n$. $\Gamma(\cdot)$ represents Euler's function.

The following lemmas will be used in this paper [2].

Lemma 1. Let $x=0$ be an equilibrium of the fractional order system:

$$
{ }_{0}^{C} D_{t}^{\alpha} x(t)=f(x) \text {. }
$$

Suppose there exist a Lyapunov function $V(t, x(t))$ and class- $k$ functions $g_{i}, i=1,2,3$, such that

$$
\begin{aligned}
g_{1}(\|x\|) & \leq V(t, x(t)) \leq g_{2}(\|x\|), \\
{ }_{0}^{C} D_{t}^{\beta} V(t, x(t)) & \leq-g_{3}(\|x\|),
\end{aligned}
$$

where $0<\beta<1$; then the equilibrium point of system (2) is Mittag-Leffler stable.

Lemma 2. Mittag-Leffler stability means asymptotical stability.

It should be pointed out that, in integer-order nonlinear systems, Lyapunov direct method (also called the Lyapunov second method) offers a method to analyze the stability of the closed-loop system without solving the differential equations explicitly. Although the Lyapunov stability theory for integer-order systems was proposed in 1892 and it has been studied and modified by lots of expert researchers, the Lyapunov stability theory for fractional order systems has been developed until recently (see Lemmas 1 and 2). Besides, we can see that the structure of stability theory for fractional order systems is very similar to the integer-order systems.

\section{Main Results}

3.1. Synchronization of Fractional Order Chaotic Systems with System Uncertainty. Consider the following master fractional order chaotic system:

$$
{ }_{0}^{C} D_{t}^{\alpha} x(t)=A x(t)+f(x(t)),
$$

where $x(t)$ represents the state vector, $A \in R^{n \times n}$ is a constant matrix, $f(x(t)) \in R^{n}$ is an unknown nonlinear function, and $\alpha \in(0,1)$ is the fractional order.

The following assumption is needed in this paper.

Assumption 3. The unknown nonlinear function $f(x(t)) \in$ $R^{n}$ is smooth enough such that $f(x(t))$ has arbitrary order derivative. Then, we can further assume that $f(x(t))$ satisfies the Lipschitz condition with respect to $x(t)$ : that is,

$$
\|f(x(t))-f(\widehat{x}(t))\| \leq l\|x(t)-\widehat{x}(t)\|,
$$

where $l>0$ is the Lipschitz constant.

Remark 4. Assumption 3 is not restrictive, since there are lots of fractional order chaotic systems satisfying Assumption 3, for example, fractional order chaotic neural networks [30], fractional order financial chaotic system [33], fractional order 
Lorenz system [34], fractional order Chen system [35], and fractional order Chua's system [36]. In fact, this assumption enables us to have a simpler analysis of the system stability.

Let us construct the following slave chaotic system:

$$
{ }_{0}^{C} D_{t}^{\alpha} \hat{x}(t)=A \hat{x}(t)+f(\widehat{x}(t))+u(t) .
$$

Define the synchronization error as

$$
e(t)=x(t)-\hat{x}(t) .
$$

Then the synchronization error dynamic equation can be given as

$$
{ }_{0}^{C} D_{t}^{\alpha} e(t)=A e(t)-f(\widehat{x}(t))+f(x(t))-u(t) .
$$

The objective of this section is to design a controller such that the slave chaotic system (7) synchronizes chaotic system (5): that is, $\lim _{t \rightarrow \infty} e(t)=0$. To meet this objective, let us construct the following robust controller:

$$
u(t)=B e(t)+u_{r}
$$

where $u_{r}$ is a robust term to cancel the unknown nonlinear function. $B \in R^{n \times n}$ is a proper constant matrix to be chosen such that

$$
A-B=-K
$$

where $K=\operatorname{diag}\left(\left[k_{1}, k_{2}, \ldots, k_{n}\right]\right)$ with $k_{i}>0, i=1,2, \ldots, n$.

Substituting controller (10) into (9), we have

$$
{ }_{0}^{C} D_{t}^{\alpha} e(t)=-K e(t)-f(\widehat{x}(t))+f(x(t))-u_{r}(t) .
$$

In the stability analysis of fractional order nonlinear systems, the Lyapunov function candidate $V(t)=e^{T}(t) e(t)$ is often used. The $\alpha$ th-order of $V(t)$ can be given as

$$
{ }_{0}^{C} D_{t}^{\alpha} V(t)=\left({ }_{0}^{C} D_{t}^{\alpha} e(t)\right)^{T} e(t)+e^{T}(t){ }_{0}^{C} D_{t}^{\alpha} e(t)+2 \Lambda,
$$

where

$$
\Lambda=\sum_{i=1}^{\infty} \frac{\Gamma(1+\alpha)}{\Gamma(1+i) \Gamma(1-i+\alpha)}{ }_{0}^{C} D_{t}^{i} e(t){ }_{0}^{C} D_{t}^{\alpha-i} e(t) .
$$

Remark 5. To achieve the results by using the stability theorems of fractional order systems, in the literatures [16, 37], the authors suppose that the bounded condition $|\Lambda| \leq$ $b|x(t)|$ holds, where $b$ is a positive constant. This condition is only verified through simulation results. But in this paper, we will give a strict proof of this condition and establish a fundamental result. This result is given to use for stability analysis of fractional order nonlinear systems, especially for Mittag-Leffler stability [2] analysis of fractional order nonlinear systems.

Lemma 6. Suppose that Assumption 3 is satisfied. Then there exists some positive constant $a>0$ such that

$$
\begin{aligned}
& \left\|\sum_{i=1}^{\infty} \frac{\Gamma(1+\alpha)}{\Gamma(1+i) \Gamma(1-i+\alpha)}{ }_{0}^{C} D_{t}^{i} e(t){ }_{0}^{C} D_{t}^{\alpha-i} e(t)\right\| \\
& \quad \leq a\|e(t)\| .
\end{aligned}
$$

Proof. From Assumption 3 we know ${ }_{0}^{C} D_{t}^{k} e(t)$ exist. It is obvious that ${ }_{0}^{C} D_{t}^{k} e(t)$ are bounded. As a result, there exists $M$ such that

$$
\left\|{ }_{0}^{C} D_{t}^{k} e(t)\right\| \leq M
$$

On the other hand, because $0<\alpha<1$, we have

$$
\left\|{ }_{0}^{C} D_{t}^{\alpha-k} e(t)\right\| \leq \bar{K}\|e(t)\|
$$

where $\bar{K}$ is a positive constant.

It is known that the gamma function is nonzero everywhere along the real line, and there is in fact no complex number $z$ for which $\Gamma(z)=0$. As a result the reciprocal gamma function $1 / \Gamma(z)$ is an entire function. There exists a lower bound $L$ such that $0<L \leq|\Gamma(1-\alpha+i)|$ for $i=1,2,3, \ldots$.

Since $\Gamma(i) / \Gamma(i+1)=1 / i$, the infinite series $\sum_{i=1}^{\infty}(1 / \Gamma(i+1))$ is convergence. So, there exists an upper bound $H>0$ such that

$$
0<\sum_{i=1}^{\infty} \frac{1}{\Gamma(i+1)}<H .
$$

From the above discussion, we can obtain the following inequality:

$$
\begin{aligned}
& \left\|\sum_{i=1}^{\infty} \frac{\Gamma(1+\alpha)}{\Gamma(1+i) \Gamma(1-i+\alpha)}{ }_{0}^{C} D_{t}^{i} e(t){ }_{0}^{C} D_{t}^{\alpha-i} e(t)\right\| \\
& \quad \leq a\|e(t)\|
\end{aligned}
$$

in which

$$
a=\frac{\Gamma(1+\alpha) M \bar{K} H}{L} .
$$

This completes the proof.

Remark 7. It should be pointed out that the value of $a$ in Lemma 6 is hard to be estimated. In this paper, we only estimate its value through simulation results.

From the above discussions, now we are ready to give the following theorem.

Theorem 8. Consider the master chaotic system (5) and the slave chaotic system (7). Suppose that Assumption 3 is satisfied. If the control input is chosen as (10), and the robust term $u_{r}(t)$ is constructed as

$$
u_{r}(t)=r \frac{e(t)}{\|e(t)\|}
$$

where $r$ is a positive constant, then synchronization between systems (5) and (7) will be achieved.

Proof. From (21) and (12) we have

$$
{ }_{0}^{C} D_{t}^{\alpha} e(t)=-K e(t)+f(x(t))-f(\widehat{x}(t))-r \frac{e(t)}{\|e(t)\|} .
$$


Let us define the Lyapunov function candidate as

$$
V(t)=e^{T}(t) e(t) .
$$

Then we have

$$
\begin{aligned}
& { }_{0}^{C} D_{t}^{\alpha} V(t)=\left({ }_{0}^{C} D_{t}^{\alpha} e(t)\right)^{T} e(t) \\
& +\sum_{i=1}^{\infty} \frac{\Gamma(1+\alpha)}{\Gamma(1+i) \Gamma(1-i+\alpha)}\left({ }_{0}^{C} D_{t}^{i} e(t)\right)\left({ }_{0}^{C} D_{t}^{\alpha-i} e(t)\right) \\
& =-e^{T}(t) K e(t)+\Lambda-r\|e(t)\| \\
& +(f(x(t))-f(\hat{x}(t)))^{T} e(t) .
\end{aligned}
$$

From Assumption 3 and Lemma 6 we have

$$
\begin{aligned}
{ }_{0}^{C} D_{t}^{\alpha} V(t) \leq & -e^{T}(t) K e(t) \\
& +\|e(t)\|\|f(x(t))-f(\widehat{x}(t))\|+\|\Lambda\| \\
& -r\|e(t)\| \\
\leq & -e^{T}(t) K e(t)+l\|e(t)\|^{2} \\
& +(a-r)\|e(t)\| .
\end{aligned}
$$

If we can choose large enough $r$ and $k$ such that $r>a$, $k>l$, where $k=\min \left\{k_{1}, k_{2}, \ldots, k_{n}\right\}$, we obtain

$$
\begin{aligned}
{ }_{0}^{C} D_{t}^{\alpha} V(t) & \leq-e^{T}(t) K e(t)+l\|e(t)\|^{2} \\
& \leq-(k-l)\|e(t)\|^{2}=-(k-l) V(t) .
\end{aligned}
$$

Form Lemmas 1 and 2, we know the synchronization error $e(t)$ will converge to zero asymptotically. And this ends the proof.

3.2. Synchronization of Fractional Order Chaotic Systems with Both System Uncertainty and Norm Bounded External Disturbance. Consider the following master and slave fractional order chaotic systems:

$$
\begin{aligned}
& { }_{0}^{C} D_{t}^{\alpha} x(t)=A x(t)+f(x(t)), \\
& { }_{0}^{C} D_{t}^{\alpha} \widehat{x}(t)=A \widehat{x}(t)+f(\widehat{x}(t))+g(t)+u(t),
\end{aligned}
$$

where $x(t)$ represents the state variables and $A \in R^{n \times n}$ is a constant matrix. $f(x(t)) \in R^{n}$ is an unknown nonlinear function which satisfies Assumption 3. $\alpha \in(0,1)$ is the fractional order.

Assumption 9. The nonlinear term $g(t)$ represents external disturbance which is smooth enough and satisfies

$$
\|g(t)\| \leq \rho(t)
$$

where $\rho(t)$ is a known positive function.
Theorem 10. Consider the master chaotic system (27) and the slave chaotic system (28). Suppose that Assumptions 3 and 9 are satisfied. If the control input is chosen as

$$
u(t)=B e(t)+r \frac{e(t)}{\|e(t)\|}+\rho(t) \frac{e(t)}{\|e(t)\|},
$$

where $r$ is a positive constant, then synchronization between systems (27) and (28) will be achieved.

Proof. From (27) and (28) we can obtain

$$
\begin{aligned}
{ }_{0}^{C} D_{t}^{\alpha} e(t)= & A e(t)+f(\widehat{x}(t))-f(x(t))-g(t) \\
& -u(t) .
\end{aligned}
$$

Substituting controller (30) into (31) yields

$$
\begin{aligned}
{ }_{0}^{C} D_{t}^{\alpha} e(t)= & -K e(t)+f(\widehat{x}(t))-f(x(t))-g(t) \\
& -r \frac{e(t)}{\|e(t)\|}-\rho(t) \frac{e(t)}{\|e(t)\|} .
\end{aligned}
$$

Defining the Lyapunov function candidate as $V(t)=$ $e^{T}(t) e(t)$, we have

$$
\begin{aligned}
& { }_{0}^{C} D_{t}^{\alpha} V(t)=\left({ }_{0}^{C} D_{t}^{\alpha} e(t)\right)^{T} e(t) \\
& +\sum_{k=1}^{\infty} \frac{\Gamma(1+\alpha)}{\Gamma(1+i) \Gamma(1-i+\alpha)}\left({ }_{0}^{C} D_{t}^{i} e(t)\right)\left({ }_{t_{0}}^{C} D_{t}^{\alpha-i} e(t)\right) \\
& =-e^{T}(t) K e(t)+(f(x(t))-f(\widehat{x}(t)))^{T} e(t) \\
& -g^{T}(t) e(t)+\Lambda-r\|e(t)\|-\rho(t)\|e(t)\| .
\end{aligned}
$$

According to Assumptions 3 and 9 and Lemma 6 we have

$$
\begin{aligned}
{ }_{0}^{C} D_{t}^{\alpha} V(t) \leq & -e^{T}(t) K e(t)+\|g(t, x(t))\|\|e(t)\| \\
& +\|e(t)\|\|f(x(t))-f(\widehat{x}(t))\|+\|\Lambda\| \\
& -r\|e(t)\|-\rho(t)\|e(t)\| \\
\leq & -e^{T}(t) K e(t)+l\|e(t)\|^{2} \\
& +(a-r)\|e(t)\| .
\end{aligned}
$$

As the same analysis in Theorem 8, if we can choose large enough $r$ and $k$, then synchronization between systems (27) and (28) will be achieved. This ends the proof of Theorem 10. 


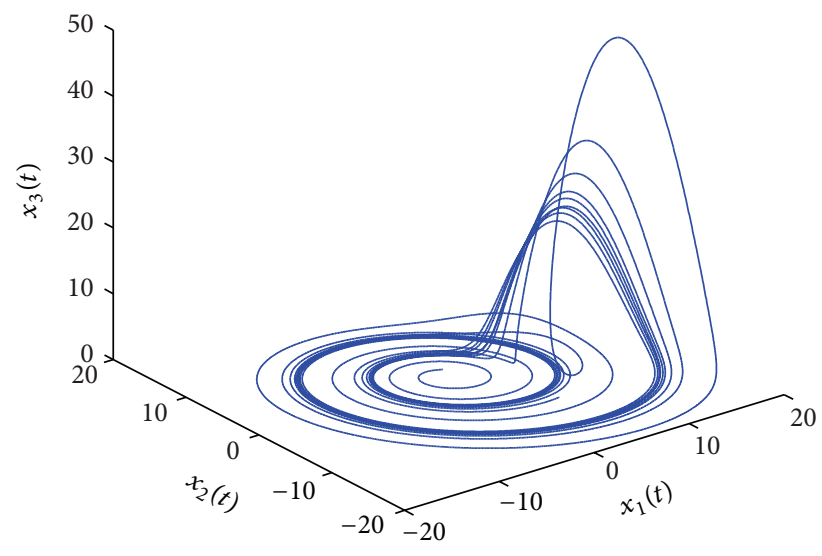

FIGURE 1: Chaotic behavior of fractional order Rössler system.

\section{Simulation Studies}

In simulation, the fractional order Rössler chaotic system will be used. The fractional order model of the Rössler system is described by

$$
\begin{aligned}
{ }_{0}^{C} D_{t}^{\alpha} x(t)= & {\left[\begin{array}{ccc}
0 & -1 & -1 \\
1 & b & 0 \\
0 & 0 & -c
\end{array}\right] x(t) } \\
& +\left[\begin{array}{c}
0 \\
0 \\
d+x_{1}(t) x_{3}(t)
\end{array}\right],
\end{aligned}
$$

where $b, c, d$ are system parameters. When $b=0.5, c=10$, $d=0.2$, and the fractional order $\alpha=0.9$, system (35) has a chaotic attractor, which is depicted in Figure 1.

Let us define the slave system as (7). The initial condition is set as $\hat{x}(0)=[-0.5,-2,2]^{T}$. From the simulation we know that the state of fractional order Rössler system is bounded, and the Lipchitz constant $l$ can be chosen as 10: that is,

$$
\|f(x(t))-f(\widehat{x}(t))\| \leq 10\|x(t)-\widehat{x}(t)\|,
$$

which means that Assumption 3 is satisfied.

From (35) we know $A=\left[\begin{array}{ccc}0 & -1 & -1 \\ 1 & 0.5 & 0 \\ 0 & 0 & -10\end{array}\right]$; then we can choose the matrix $B$ as $B=\left[\begin{array}{ccc}12 & -1 & -1 \\ 1 & 12.5 & 0 \\ 0 & 0 & 2\end{array}\right]$. As a result, $-K=\operatorname{diag}([12,12,12])$, which means the condition $k>$ $l$ in Theorem 8 is satisfied. According to Theorem 8 , the synchronization between master and slave systems can be achieved. The parameter $r$ of the robust term is selected as $r=0.5$. The simulation results are shown in Figures 25. From Figure 2, we can see a quick convergence of the synchronization errors. Figures 3-5 show the boundedness of the control inputs.

To show the robustness of the proposed control method, we consider the following external disturbance in slave system (28):

$$
g(t)=\left[\sin (t), \cos (t), \frac{1}{2} \sin ^{2}(t)\right]^{T} .
$$

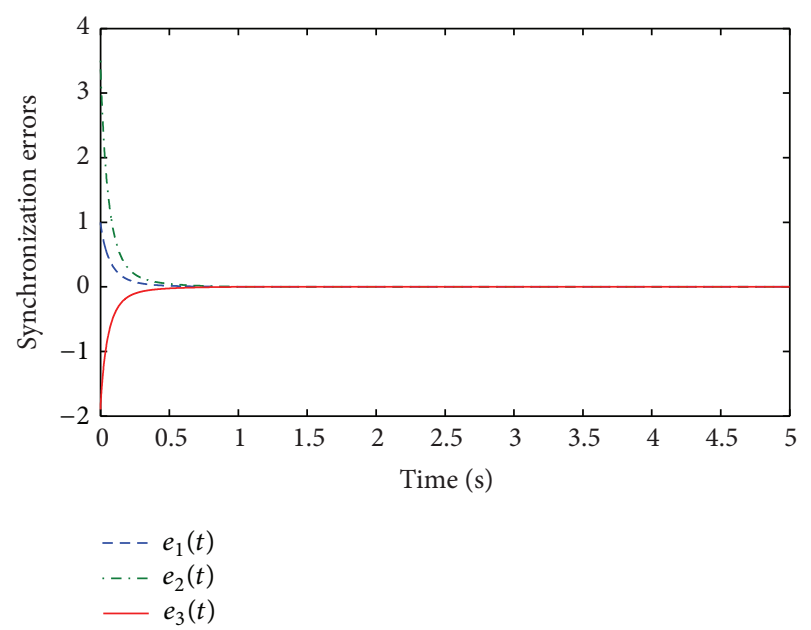

FIGURE 2: Time response of synchronization errors.

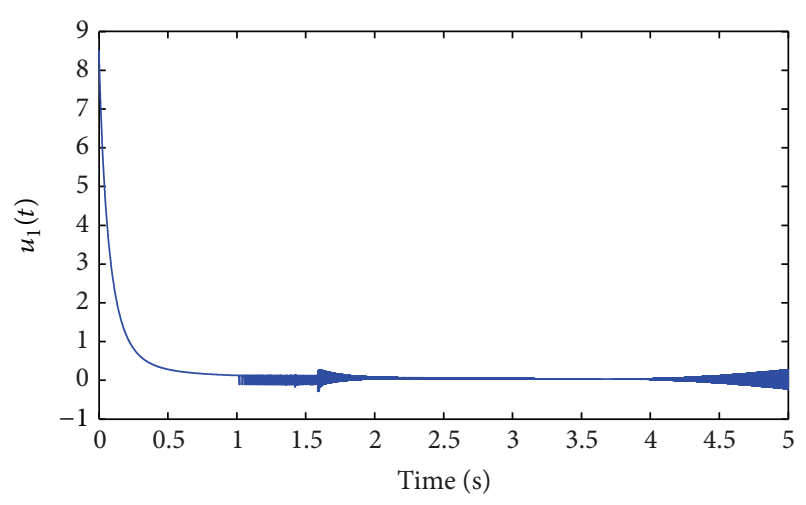

FIGURE 3: Time response of control input $u_{1}(t)$.

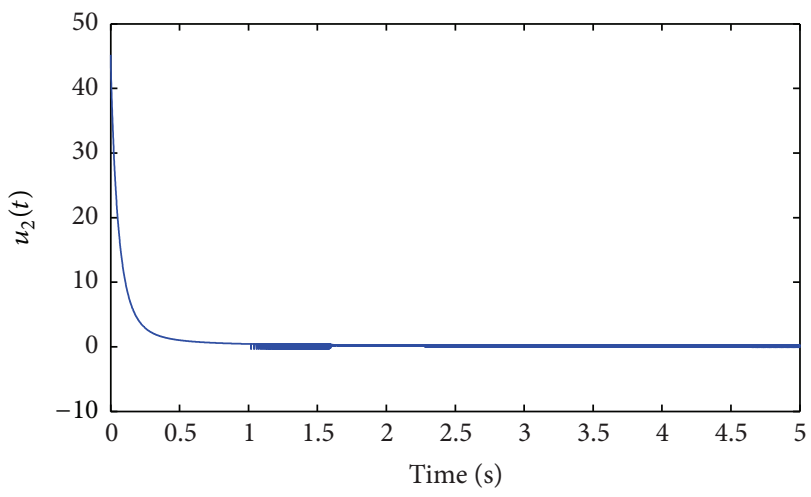

Figure 4: Time response of control input $u_{2}(t)$.

Then we can choose $\rho(t)$ as $\rho(t)=1.5$. Then we can declare that $\|g(t)\| \leq \rho(t)$, which means that Assumption 9 is satisfied. According to Theorem 10 we know that synchronization between systems (27) and (28) can be achieved. The simulation results are presented in Figures 6-9. From the simulation results we can conclude that good synchronization 


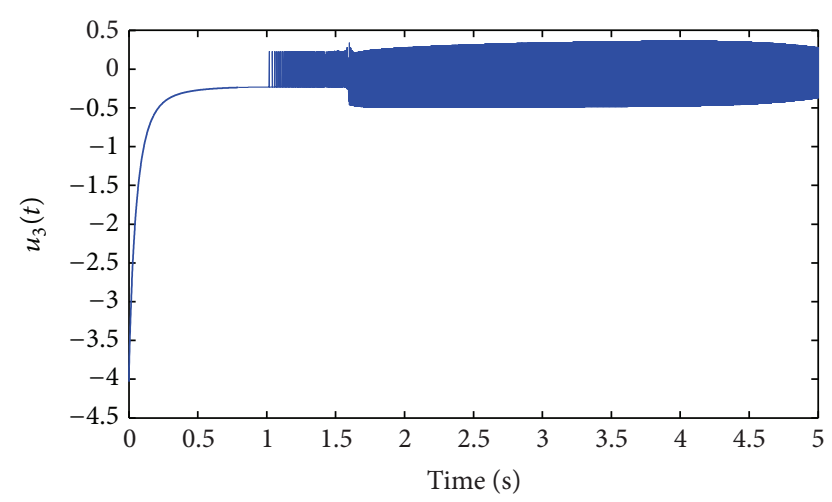

Figure 5: Time response of control input $u_{3}(t)$.

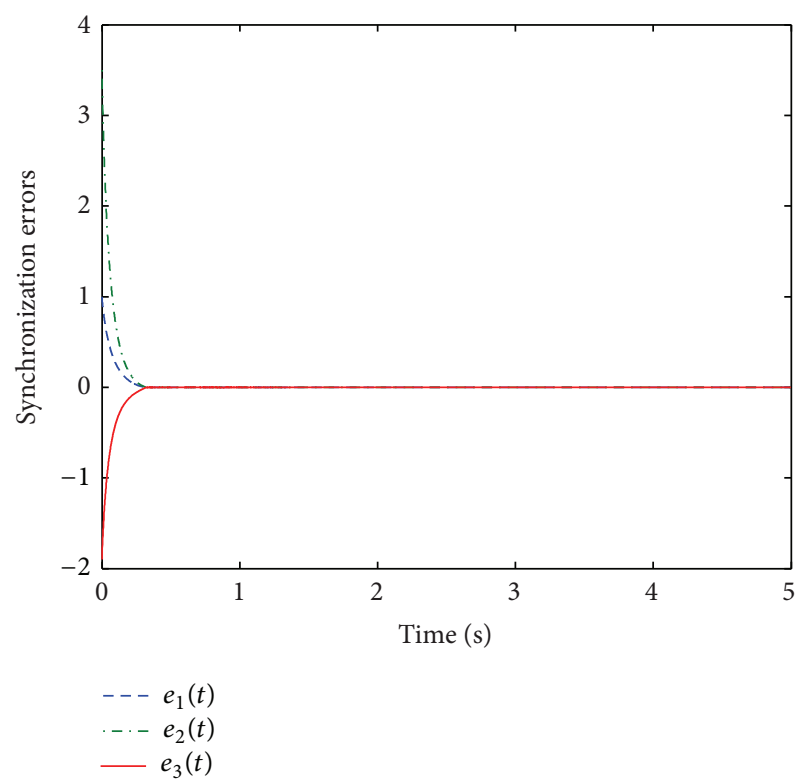

FIGURE 6: Simulation results with external disturbance: time response of synchronization errors.

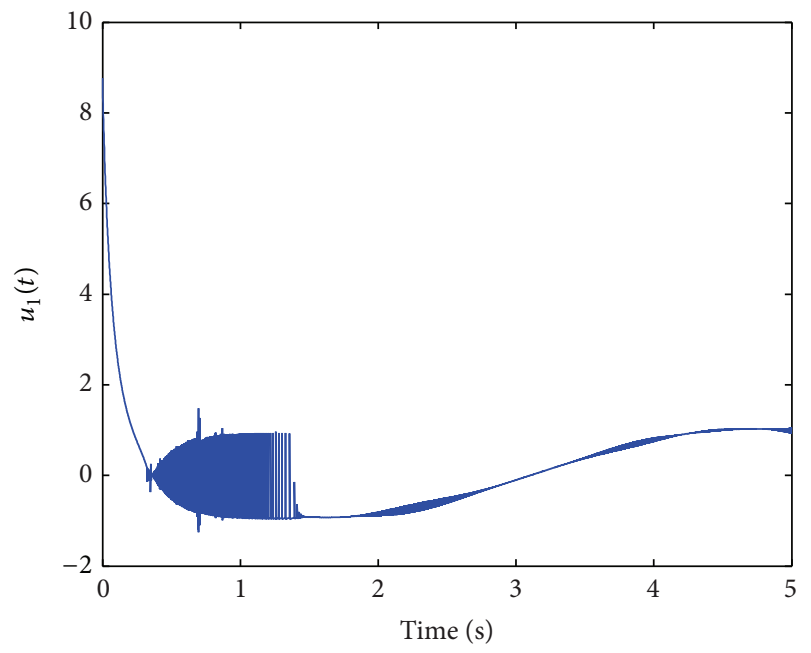

FIGURE 7: Simulation results with external disturbance: control input $u_{1}(t)$.

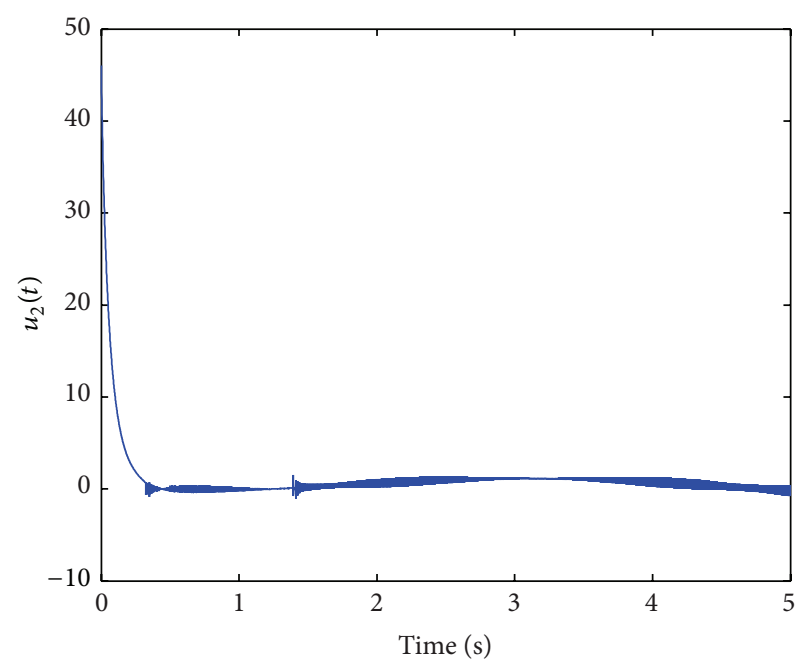

FIGURE 8: Simulation results with external disturbance: control input $u_{2}(t)$.

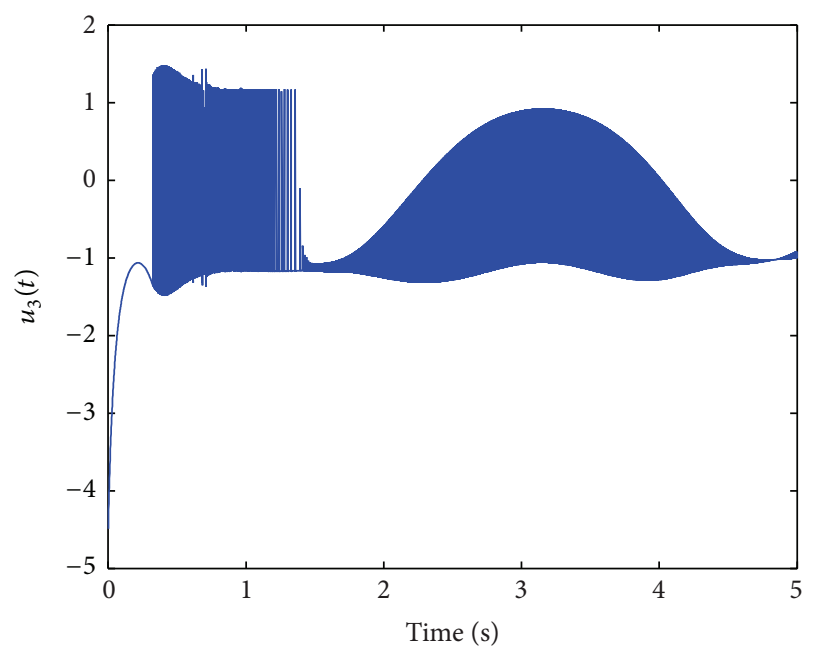

FIgURE 9: Simulation results with external disturbance: control input $u_{3}(t)$.

performance has been obtained. We can see that, in simulation results, the chattering phenomenon does exist because of the sign function in controllers (21) and (30).

\section{Conclusion}

We investigate the robust synchronization of fractional order chaotic systems with both system uncertainties and norm bounded external disturbances. A boundary condition, which is introduced by differentiating the squared Lyapunov function with fractional order, is verified. The boundary condition enables us to have a simpler analysis of system stability by using Lyapunov directed method. It is shown that the synchronization errors converge to zero asymptotically. The effectiveness of the proposed method is confirmed by two numerical simulations. How to cancel the chattering 
phenomenon in the synchronization controller design is one of our investigation directions.

\section{Conflict of Interests}

The authors do not have a direct financial relation with any commercial identity mentioned in their paper that might lead to a conflict of interests for any of the authors.

\section{Acknowledgments}

This research is supported by the National Natural Science Foundation of China (Grant no. 11401008), the Natural Science Foundation for the Higher Education Institutions of Anhui Province of China (Grant nos. KJ2015A256 and KJ2014A064), the Natural Science Foundation for Anhui University of Science and Technology (Grant no. 201428), and the Natural Science Foundation for Huainan Normal University (Grant no. 2015XJ07ZD).

\section{References}

[1] I. Podlubny, Fractional Differential Equations, vol. 198 of Mathematics in Science and Engineering, Academic Press, New York, NY, USA, 1999.

[2] Y. Li, Y. Q. Chen, and I. Podlubny, "Mittag-Leffler stability of fractional order nonlinear dynamic systems," Automatica, vol. 45, no. 8, pp. 1965-1969, 2009.

[3] X.-J. Wen, Z.-M. Wu, and J.-G. Lu, "Stability analysis of a class of nonlinear fractional-order systems," IEEE Transactions on Circuits and Systems II: Express Briefs, vol. 55, no. 11, pp. 11781182, 2008.

[4] J. G. Liu, L. H. Huang, and Y. M. Meng, "Adaptive synchronization of fractional-order complex networks via pinning control," International Journal of Adaptive Control and Signal Processing, vol. 27, no. 12, pp. 1086-1096, 2013.

[5] D.-F. Wang, J.-Y. Zhang, and X.-Y. Wang, "Robust modified projective synchronization of fractional-order chaotic systems with parameters perturbation and external disturbance," Chinese Physics B, vol. 22, no. 10, Article ID 100504, 2013.

[6] J. H. Koo, D. H. Ji, and S. C. Won, "Synchronization of singular complex dynamical networks with time-varying delays," Applied Mathematics and Computation, vol. 217, no. 8, pp. 39163923, 2010.

[7] J. W. Wang and Y. B. Zhang, "Network synchronization in a population of star-coupled fractional nonlinear oscillators," Physics Letters A, vol. 374, no. 13-14, pp. 1464-1468, 2010.

[8] Y. Yang and G. P. Chen, "Finite-time stability of fractional order impulsive switched systems," International Journal of Robust and Nonlinear Control, vol. 25, no. 13, pp. 2207-2222, 2015.

[9] G. Wen, W. Yu, G. Hu, J. Cao, and X. Yu, "Pinning synchronization of directed networks with switching topologies: a multiple Lyapunov functions approach," IEEE Transactions on Neural Networks and Learning Systems, vol. 26, no. 12, pp. 3239-3250, 2015.

[10] S. Djennoune and M. Bettayeb, "Optimal synergetic control for fractional-order systems," Automatica, vol. 49, no. 7, pp. 22432249, 2013.

[11] H. Liu, S. Li, Y. Sun, and H. Wang, "Adaptive fuzzy synchronization for uncertain fractional-order chaotic systems with unknown non-symmetrical control gain," Acta Physica Sinaca, vol. 64, no. 7, Article ID 070503, 2015.

[12] H. Liu, S. Li, Y. Sun, and H. Wang, "Prescribed performance synchronization for fractional-order chaotic systems," Chinese Physics B, vol. 24, no. 9, Article ID 090505, 2015.

[13] S. Kamal, A. Raman, and B. Bandyopadhyay, "Finite-time stabilization of fractional order uncertain chain of integrator: an integral sliding mode approach," IEEE Transactions on Automatic Control, vol. 58, no. 6, pp. 1597-1602, 2013.

[14] J. Shen and J. Lam, "Non-existence of finite-time stable equilibria in fractional-order nonlinear systems," Automatica, vol. 50, no. 2, pp. 547-551, 2014.

[15] J. Sabatier, C. Farges, M. Merveillaut, and L. Feneteau, "On observability and pseudo state estimation of fractional order systems," European Journal of Control, vol. 18, no. 3, pp. 260271, 2012.

[16] S. Dadras and H. R. Momeni, "Fractional sliding mode observer design for a class of uncertain fractional order nonlinear systems," in Proceedings of the 50th IEEE Conference on Decision and Control and European Control Conference (CDC-ECC '11), pp. 6925-6930, IEEE, Orlando, Fla, USA, December 2011.

[17] J. C. Trigeassou, N. Maamri, J. Sabatier, and A. Oustaloup, "State variables and transients of fractional order differential systems," Computers and Mathematics with Applications, vol. 64, no. 10, pp. 3117-3140, 2012.

[18] S. Kuntanapreeda, "Robust synchronization of fractional-order unified chaotic systems via linear control," Computers and Mathematics with Applications, vol. 63, no. 1, pp. 183-190, 2012.

[19] G. Si, Z. Sun, Y. Zhang, and W. Chen, "Projective synchronization of different fractional-order chaotic systems with nonidentical orders," Nonlinear Analysis: Real World Applications, vol. 13, no. 4, pp. 1761-1771, 2012.

[20] G. Garcia, S. Tarbouriech, and J. Bernussou, "Finite-time stabilization of linear time-varying continuous systems," IEEE Transactions on Automatic Control, vol. 54, no. 2, pp. 364-369, 2009.

[21] G. H. Wen, Z. Duan, W. Yu, and G. Chen, "Consensus in multiagent systems with communication constraints," International Journal of Robust and Nonlinear Control, vol. 22, no. 2, pp. 170$182,2012$.

[22] G. H. Wen, Z. S. Duan, W. W. Yu, and G. R. Chen, "Consensus of multi-agent systems with nonlinear dynamics and sampleddata information: a delayed-input approach," International Journal of Robust and Nonlinear Control, vol. 23, no. 6, pp. 602-619, 2013.

[23] G. H. Wen, Z. S. Duan, G. R. Chen, and W. W. Yu, "Consensus tracking of multi-agent systems with Lipschitz-type node dynamics and switching topologies," IEEE Transactions on Circuits and Systems I: Regular Papers, vol. 61, no. 2, pp. 499-511, 2014.

[24] J. Wang, Z. Duan, G. Wen, and G. Chen, "Distributed robust control of uncertain linear multiagent systems," International Journal of Robust and Nonlinear Control, vol. 25, no. 13, pp. 21622179, 2015.

[25] F. Zhi, G. Hu, and G. Wen, "Distributed consensus tracking for multi-agent systems under two types of attacks," International Journal of Robust and Nonlinear Control, 2015.

[26] Y. D. Ma, J. G. Lu, and W. D. Chen, "Robust stability and stabilization of fractional order linear systems with positive real uncertainty," ISA Transactions, vol. 53, no. 2, pp. 199-209, 2014. 
[27] N. Tan, Ö. F. Özgüven, and M. M. Özyetkin, "Robust stability analysis of fractional order interval polynomials," ISA Transactions, vol. 48, no. 2, pp. 166-172, 2009.

[28] F. Amato, C. Cosentino, and A. Merola, "Sufficient conditions for finite-time stability and stabilization of nonlinear quadratic systems," IEEE Transactions on Automatic Control, vol. 55, no. 2, pp. 430-434, 2010.

[29] S. H. Hosseinnia, R. Ghaderi, A. Ranjbar, N. M. Mahmoudian, and S. Momani, "Sliding mode synchronization of an uncertain fractional order chaotic system," Computers \& Mathematics with Applications, vol. 59, no. 5, pp. 1637-1643, 2010.

[30] H. Liu, S. Li, H. Wang, Y. Huo, and J. Luo, "Adaptive synchronization for a class of uncertain fractional-order neural networks," Entropy, vol. 17, no. 10, pp. 7185-7200, 2015.

[31] S. H. Hosseinnia, R. Ghaderi, A. N. Ranjbar, M. Mahmoudian, and S. Momani, "Sliding mode synchronization of an uncertain fractional order chaotic system," Computers and Mathematics with Applications, vol. 59, no. 5, pp. 1637-1643, 2010.

[32] C. Yin, S. Dadras, and S.-m. Zhong, "Design an adaptive sliding mode controller for drive-response synchronization of two different uncertain fractional-order chaotic systems with fully unknown parameters," Journal of the Franklin Institute, vol. 349, no. 10, pp. 3078-3101, 2012.

[33] W.-C. Chen, "Nonlinear dynamics and chaos in a fractionalorder financial system," Chaos, Solitons \& Fractals, vol. 36, no. 5, pp. 1305-1314, 2008.

[34] X.-Y. Wang and J.-M. Song, "Synchronization of the fractional order hyperchaos Lorenz systems with activation feedback control," Communications in Nonlinear Science and Numerical Simulation, vol. 14, no. 8, pp. 3351-3357, 2009.

[35] C. Li and G. Chen, "Chaos in the fractional order Chen system and its control," Chaos, Solitons \& Fractals, vol. 22, no. 3, pp. 549-554, 2004.

[36] T. T. Hartley, C. F. Lorenzo, and H. K. Qammer, "Chaos in a fractional order Chua's system," IEEE Transactions on Circuits and Systems I: Fundamental Theory and Applications, vol. 42, no. 8, pp. 485-490, 1995.

[37] M. Ö. Efe, "Fractional fuzzy adaptive sliding-mode control of a 2-DOF direct-drive robot arm," IEEE Transactions on Systems, Man, and Cybernetics, Part B: Cybernetics, vol. 38, no. 6, pp. 1561-1570, 2008. 


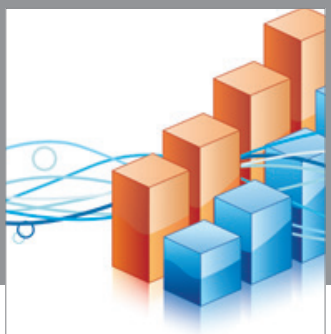

Advances in

Operations Research

vatem alat4

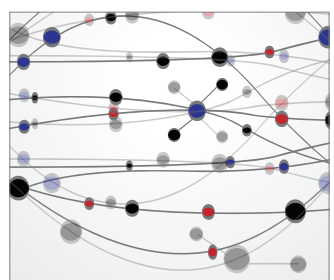

\section{The Scientific} World Journal
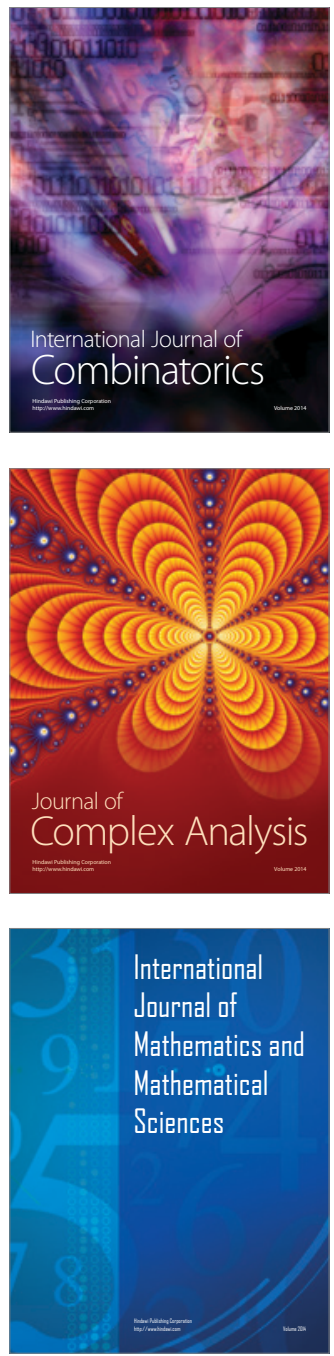
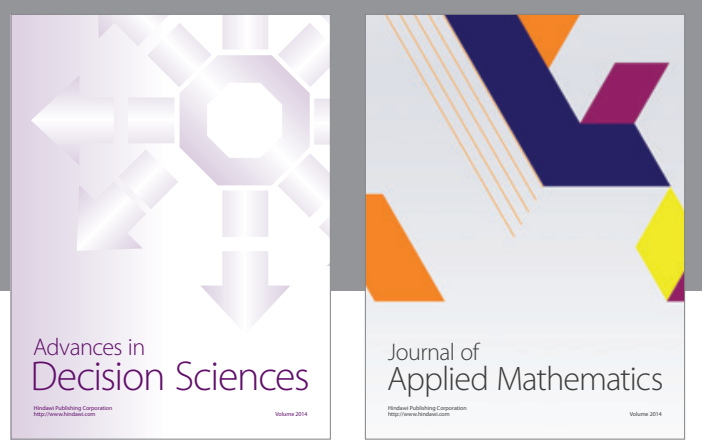

Algebra

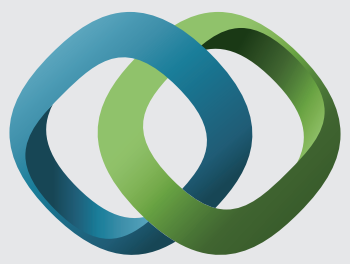

\section{Hindawi}

Submit your manuscripts at

http://www.hindawi.com
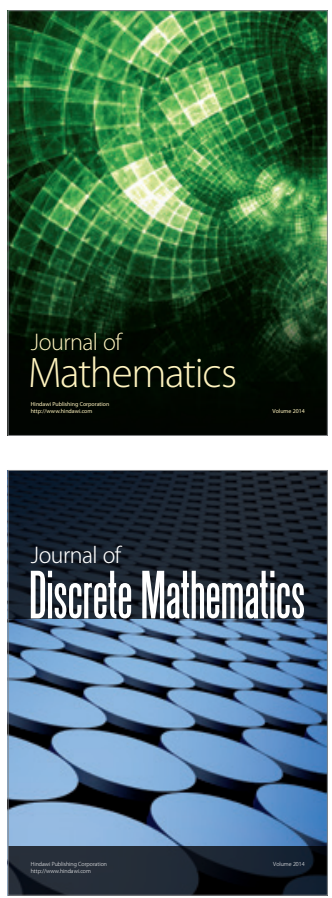

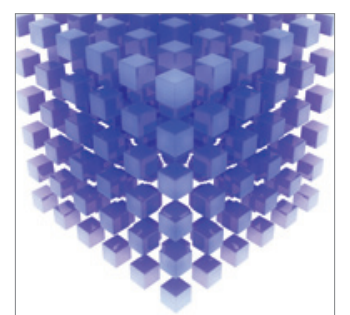

Mathematical Problems in Engineering
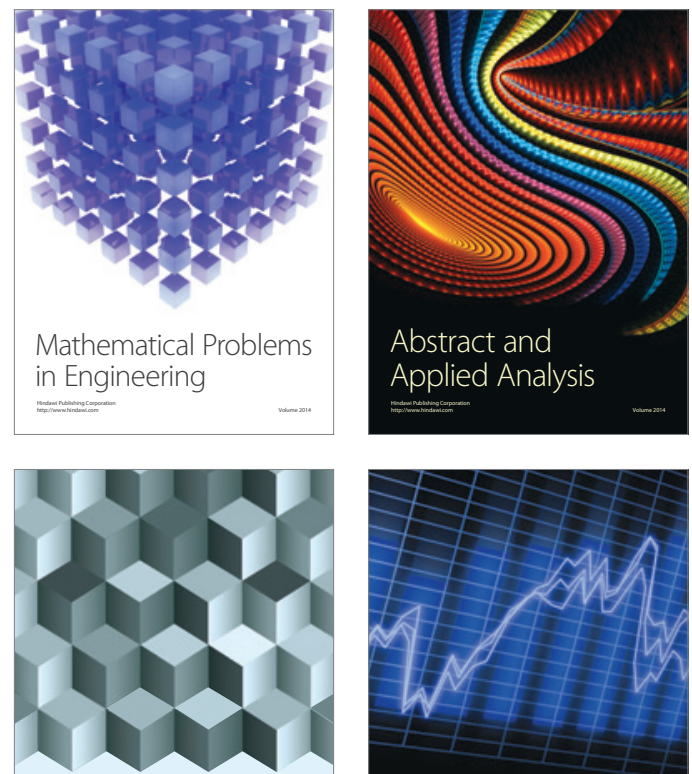

Journal of

Function Spaces

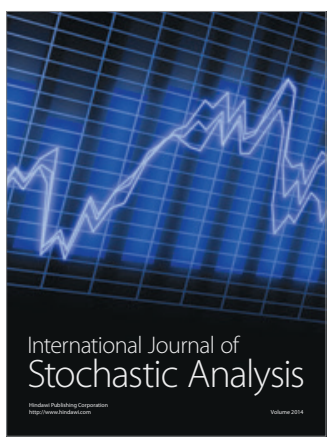

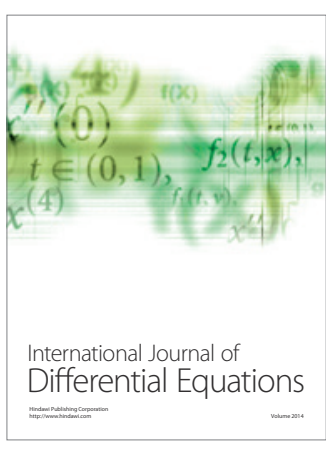
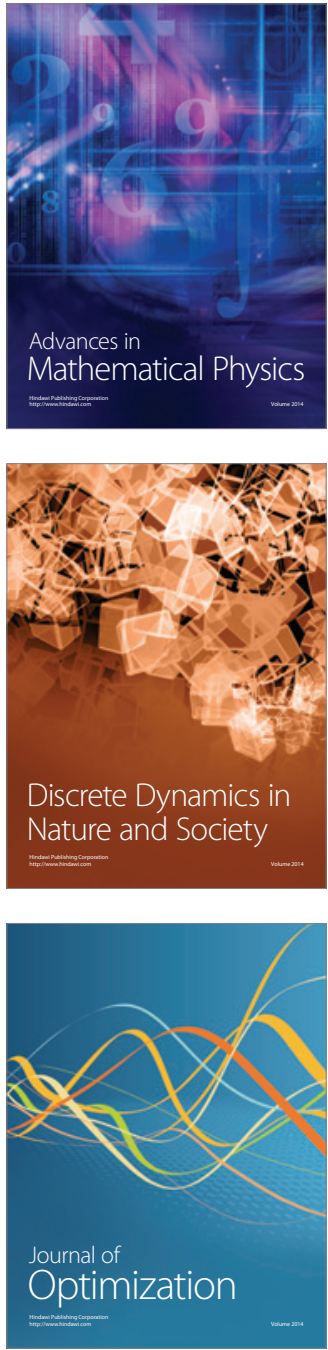